\title{
Clinical Pathological Features of Peripheral T Cell Lymphoma with Concurrent Bone Marrow Involvement
}

\author{
Mohamed Masoud, Catherine Xie, Jessica Zhou, Xia Chen, Ming Xie ${ }^{*}$ \\ Department of Pathology, Oakland University William Beaumont School of Medicine, Troy, USA \\ Email address: \\ ming.xie@beaumont.org (Ming Xie) \\ ${ }^{*}$ Corresponding author \\ To cite this article: \\ Mohamed Masoud, Catherine Xie, Jessica Zhou, Xia Chen, Ming Xie. Clinical Pathological Features of Peripheral T Cell Lymphoma with \\ Concurrent Bone Marrow Involvement. American Journal of Clinical and Experimental Medicine. Vol. 6, No. 1, 2018, pp. 22-26. \\ doi: 10.11648/j.ajcem.20180601.14
}

Received: December 11, 2017; Accepted: January 10, 2018; Published: January 29, 2018

\begin{abstract}
Peripheral T cell lymphomas (PTCLs) are uncommon and less well studied. Most PTCLs present as systemic disease and often involve bone marrow. Bone marrow involvement by PTCLs may damage the normal hematopoiesis and bring more challenge to clinical management of these patients. This study focuses on the clinical pathological features and clinical outcomes in 13 patients with nodal peripheral $\mathrm{T}$ cell lymphoma and subsequent bone marrow biopsy positive for the same lymphoma. Eight patients were diagnosed of peripheral T cell lymphoma - not otherwise specified (PTCL-NOS), 3 angioimmunoblastic T cell lymphoma (AITL), 1 anaplastic large cell lymphoma (ALCL), and $1 \mathrm{~T}$ cell lymphoma of gammadelta origin (TCL-gamma/delta). All patients had peripheral blood abnormalities: 11 anemia, 7 thrombocytopenia, 5 neutropenia, and 2 lymphocytosis. Of interest, the CD4/CD8 phenotype of PTCL was shown correlating with abnormal peripheral blood findings: CD4+/CD8- phenotype was more often associated with anemia and thrombocytopenia, and CD4/CD8+ phenotype was associated with lymphocytosis. Chemotherapy remains the choice of first line treatment for these patients with or without stem cell transplantation. 12 patients had treatment and follow up data available for review, 5 were in remission or free of disease during the follow up period; 5 patients were alive or in hospice with persistent disease and 2 died of disease due to multiple complications. The outcome of PTCL treated with the standard chemotherapy has been less favorable compared with B cell lymphomas. Majority of the patients with nodal PTCL have bone marrow involvement at the time of initial diagnosis, which has significant impact on normal hematopoiesis and may be a significant factor in the overall unfavorable prognosis for these patients. Further investigation with better knowledge about this disease will be helpful in the development of more efficient therapy and improve the disease free survival and life quality for these patients.
\end{abstract}

Keywords: Peripheral T Cell Lymphoma, Bone Marrow Biopsy, Anemia, Thrombocytopenia, Lymphocytosis

\section{Introduction}

Peripheral T cell lymphomas (PTCLs) are uncommon diseases, composing about $10 \%$ to $15 \%$ of non-Hodgkin lymphoma and about $6 \%$ of all lymphomas $[1,2]$. PTCLs are divided into nodal, extranodal, cutaneous and leukemic based on the clinical presentation and organ system involvement [3, 4]. Nodal PTCLs are the most common and usually present as late stage disease at diagnosis with spread to other organ systems including bone marrow [5, 6]. PTCL involving bone marrow is not only an indication for late stage disease, but also impact the normal hematopoietic functions and may bring more challenges for the treatment and management of these patients [7].

The treatment of PTCLs has been traditionally similar to diffuse large B cell lymphoma [8, 9]. However, the clinical outcome has been poor with the exception of low risk ALKpositive ALCL, and the standards of care for PTCLs have not been established, especially for patients with persistent disease $[10,11]$. Due to its rarity, the study on PTCLs is still limited and the clinical and pathological features of PTCL involving bone marrow are not well illustrated. This study is focused on the pathological features of these patients with clinical correlation and follow up studies. 


\section{Methods and Materials}

\subsection{Patient Selection}

The pathological reports filed at the Department of Pathology, William Beaumont Hospital - Troy from 2005 to 2016 were reviewed. In total, 13 patients were identified with PTCL in a lymph node biopsy and subsequent staging bone marrow biopsy positive for the same lymphoma.

\subsection{Routine Histological Examination of Lymph Node and Bone Marrow}

Both lymph node and bone marrow biopsy samples were processed and Hematoxylin and Eosin (H\&E) stained slides were prepared for routine microscopic examination. Each bone marrow biopsy sample included bone marrow core biopsy, bone marrow aspirate and peripheral blood. Bone marrow aspirate and peripheral blood smears were prepared with Giemsa and Wright stains for cytological evaluation.

\subsection{Flow Cytometry and Immunohistochemistry}

Portions of fresh lymph node and bone marrow aspirate were sent for flow cytometry evaluation in all cases. Multicolor flow cytometry evaluation was focused on identifying evidence of lymphoma, leukemia and myelodysplasia. A more sensitive and specific $\mathrm{T}$ cell receptor-V beta (TCR-V beta) panel was added since 2010 . Immunohistochemical studies were performed on some samples as needed with adequate controls.

\subsection{Cytogenetic, FISH and T Cell Receptor (TCR) Gene Rearrangement Studies}

All bone marrow aspirate samples were submitted for conventional cytogenetic chromosomal analysis and some samples also had myelodysplastic syndrome (MDS) FISH panel for the evaluation of cytopenia as clinically indicated. TCR gene rearrangement study by PCR was performed in the questionable samples to confirm the diagnosis of $\mathrm{T}$ cell clonality.

\subsection{Clinical Data Collection}

The electronic medical charts of these patients were retrospectively reviewed and the clinical data was collected, including clinical presentation, additional laboratory and imaging evaluations, treatment received and clinical follow up. 12 patients had therapy and follow up data available for review. The clinical follow up period was 1 to 10 years after the initial diagnosis of PTCL and bone marrow biopsy.

\section{Results}

\subsection{General Information and Pathological Diagnosis of Lymph Node Biopsy}

There were 9 males and 4 females, with median age of 60 years (44 to 83). The original diagnosis of PTCL from lymph node biopsies was listed in Table 1, including 8 PTCL-NOS, 3 AITL, 1 ALCL and 1 TCL of gamma-delta origin. All diagnosis was confirmed by flow cytometry analysis with or without immunohistochemical studies. PTCLs were further classified according to the 2008 WHO classification system [12]. In the questionable samples, the diagnosis of a $\mathrm{T}$ cell lymphoma was confirmed by positive TCR gene rearrangement studies.

Table 1. Summary of diagnosis and clinical follow up in patients with PTCLs.

Clinical follow up outcomes

\begin{tabular}{llllll}
\hline Diagnosis & Cases & Remission & With disease & Deceased & No follow up \\
\hline $\begin{array}{l}\text { PTCL- } \\
\text { NOS }\end{array}$ & 8 & 1 & 4 & 2 & 1 \\
AITL & 3 & 2 & 1 & & \\
ALCL & 1 & 1 & & & \\
TCL-g/d & 1 & 1 & & 2 & 1 \\
Total & 13 & 5 & 5 & & \\
\hline
\end{tabular}

Abbreviations: PTCL-NOS, Peripheral T cell lymphoma, not otherwise specified;

AITL, Angioimmunoblastic T cell lymphoma; ALCL, Anaplastic large cell lymphoma;

TCL-g/d, T cell lymphoma - gamma/delta origin.

\subsection{Bone Marrow Biopsy and Peripheral Blood Findings}

1. Bone marrow biopsy was performed for the staging evaluation of PTCL as well as the abnormal findings of complete blood count (CBC). The diagnosis of PTCL in bone marrow biopsies was established by histological examination and confirmed by flow cytometry, immunohistochemistry and/or $\mathrm{T}$ cell receptor gene rearrangement studies.

2. All patients had abnormal CBC findings (Figure 1). Anemia was the most common, followed by thrombocytopenia, neutropenia and lymphocytosis. Cytogenetic chromosomal analysis and FISH studies revealed normal karyotype in 11 patients, and iso $7 \mathrm{q}$ in 1 patient with TCL-gamma/delta origin and $t(5 ; 9)$ in another patient with AITL. There was no diagnosis of myelodysplasia or myelodysplasia-related changes revealed in the pathology reports by morphological, flow cytometry, cytogenetic chromosomal analysis or FISH studies in these patients. 


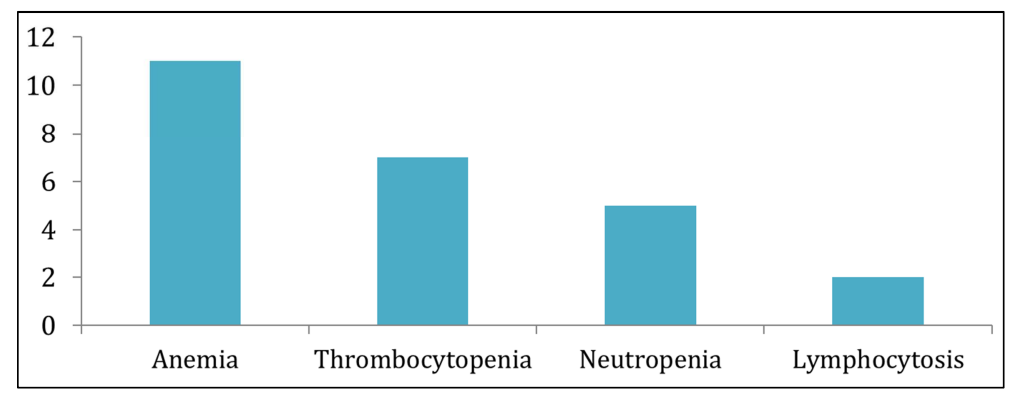

Figure 1. Abnormal CBC in patients with PTCLs.

\subsection{Correlation of CD4/CD8 Phenotype and the Abnormal CBC Findings}

Among the 13 patients in this study, 9 had PTCLs of CD4+/CD8- phenotype, 3 CD4-/CD8+ and 1 CD4-/CD8-. As shown in Table 2, all patients with PTCLs of CD4+/CD8phenotype had anemia. Thrombocytopenia was also more seen in patients with PTCLs of CD4+/CD8- phenotype. Lymphocytosis was only seen in patients with PTCLs of CD4-/CD8+ phenotype. Neutropenia was associated with both CD4+/CD8- and CD4-/CD8+ phenotypes.

Table 2. Correlation of CD4/CD8 phenotype with abnormal CBC findings.

\begin{tabular}{llll}
\hline & \multicolumn{2}{l}{ CD4/CD8 phenotype } & \\
\cline { 2 - 4 } & CD4+/CD8- & CD4-/CD8+ & CD4-/CD8- \\
\hline PTCLs & 9 & 3 & 1 \\
CBC findings & & & \\
Anemia & 9 & 1 & 1 \\
Thrombocytopenia & 6 & 1 & 0 \\
Neutropenia & 2 & 2 & 1 \\
Lymphocytosis & 0 & 2 & 0 \\
\hline
\end{tabular}

\subsection{Treatment and Clinical Follow up}

Chemotherapy and clinical follow up data was available for review in 12 patients, except for 1 patient with PTCLNOS (Table 1). Chemotherapy with CHOP regimen was the choice of first line therapy with or without stem cell transplantation. 5 patients were in remission with no evidence of lymphoma in the subsequent post-chemotherapy bone marrow biopsies (1 ALCL, 1 PTCL-NOS, 1 TCLgamma/delta and 2 AITL); 5 patients were alive or in hospice with persistent disease (1 AITL and 4 PTCL-NOS) and 2 patients died of disease (2 PTCL-NOS) due to multiple complications and infections.

\section{Discussion}

PTCL is a heterogeneous group of diseases with a diverse clinical presentation $[13,14,15]$. The majority of patients with PTCLs presents as systemic disease including bone marrow involvement [16]. In this study of nodal PTCLs with bone marrow involvement, PTCL-NOS was the most common subtype followed by AITL and ALCL, similar to the incidence of PTCL in the general population [17]. In addition to routine histological examination, immunophenotyping by flow cytometry, immunohistochemistry and occasionally TCR gene rearrangement studies are required to establish the diagnosis of
$\mathrm{T}$ cell lymphoma in a bone marrow biopsy sample.

PTCL generally affects people older than 60 years [18, 19]. The patient median age in this study was 60 years with a male predominance (9/4). All patients had abnormal laboratory $\mathrm{CBC}$ findings. Anemia was most common, followed by thrombocytopenia, neutropenia and lymphocytosis. Myelodysplasia is a common etiology for anemia or cytopenia in patients of this age group [20,21]. Evaluation of myelodysplasia in bone marrow samples was performed in these patients since it was indicated clinically for the evaluation of abnormal CBC changes of unknown etiology. There was no morphological evidence of significant myelodysplasia in the bone marrow biopsy samples, and the findings of cytogenetic chromosomal analysis and FISH studies were relatively nonspecific. These results support that the cause of abnormal $\mathrm{CBC}$ changes in these patients was likely due to PTCL involvement in bone marrow.

Of interest was the CD4/CD8 phenotype of the PTCL in this patient group apparently correlating with the abnormal peripheral blood findings. Most nodal PTCL-NOS are of Thelper phenotype (CD4+/CD8-) [3]. PTCLs with CD4+/CD8- phenotype was highly correlating with anemia and thrombocytopenia in this study. Neutropenia was seen in PTCLs of both CD4+/CD8- and CD4-/CD8+ phenotypes. But lymphocytosis was seen only in PTCLs with CD4-CD8+ phenotype. CD4+ T cells play an important role in the signal transmission of immune system and bone marrow hematopoiesis $[22,23]$. Impaired function in CD4+ $\mathrm{T}$ cells may impact the normal hematopoiesis, including erythropoiesis and thrombopoiesis [24, 25]. CD8+ T cell proliferation is likely the cause for lymphocytosis seen in peripheral blood in patients with PTCLs of CD4-/CD8+ phenotype.

Bone marrow biopsy is important in the clinical management of patients with PTCLs for both lymphoma staging and the evaluation of abnormal CBC changes. PTCL involving bone marrow is likely the cause of anemia, thrombocytopenia and neutropenia in these patients, and may have negative impact on the overall disease free survival and the life quality of these patents. Chemotherapy with or without stem cell transplantation may bring remission in some patients with PTCL. However, persistent disease is common in these patients and more efficient therapies with supportive management are needed to improve the overall clinical outcome in patients with PTCL. 


\section{Conclusion}

Although less common and not well studied, PTCLs often present as systemic disease with bone marrow involvement. All patients with PTCLs in this study had abnormal CBC findings including anemia, thrombocytopenia, neutropenia, and lymphocytosis. PTCLs with CD4+/CD8- phenotype were more often associated with anemia and thrombocytopenia, and PTCLs with CD4-/CD8+ phenotype were associated with lymphocytosis. Bone marrow involvement in patients with PTCLs may have significant negative impact on normal hematopoiesis which may be a significant factor in the overall poor prognosis in these patients. The outcome of patients with PTCLs treated with the standard chemotherapy has been less favorable and persistent disease is common. Further investigation and better knowledge about this disease are needed for more efficient therapy and improving the disease free survival and life quality for these patients.

\section{Acknowledgements}

Department of Pathology, OUWB School of Medicine, William Beaumont Hospital

\section{Abbreviations}

PTCL-NOS: Peripheral T cell lymphoma, not otherwise specified

AITL: Angioimmunoblastic T cell lymphoma

ALCL: Anaplastic large cell lymphoma

TCL-g/d: T cell lymphoma - gamma/delta origin

ALK: Anaplastic lymphoma kinase

MDS: Myelodysplastic Syndrome

CBC: Complete Blood Count

FISH: Fluorescence In Situ Hybridization

TCR: T cell receptor

PCR: Polymerase chain reaction

\section{References}

[1] International T-Cell Lymphoma Project. International Peripheral T-Cell and Natural Killer/T-Cell Lymphoma Study: pathology findings and clinical outcomes. J Clin Oncol. 2008; 26: 4124-4130.

[2] Wang SS and Vose JM. Epidemiology and Prognosis of T-Cell Lymphoma. T-Cell Lymphomas, Contemporary Hematology, 25-39 DOI 10.1007/978-1-62703-170-7_2, (C) Springer Science+Business Media New York 2013.

[3] Savage KJ. Prognosis and Primary Therapy in Peripheral TCell Lymphomas. ASH Hematology, The Education Program 2008: 280-288.

[4] Savage KJ. Aggressive peripheral T-cell lymphomas (specified and unspecified types). Hematology/the Education Program of the American Society of Hematology. 2005; 267277.

[5] Gallamini A, Stelitano C, Calvi R et al. Peripheral T-cell lymphoma unspecified (PTCL-U): a new prognostic model from a retrospective multicenter clinical study. Blood. 2004; 103 (7): 474-2479.

[6] Maricer P. Escalon MP, Liu NS, Yang Y, Hess M, Walker PL, Smith TL and Dang NH. Prognostic Factors and Treatment of Patients with T-Cell Non-Hodgkin Lymphoma: The M. D. Anderson Cancer Center Experience. Cancer 2005; 103 (10): 2091-2098.

[7] Chernova NG, Sidorova YV, Ryzhikova NV, Smirnova SY, Julhakyan HL, Sudarikov AB, Vinogradova YE, Kovrigina AM,. Zvonkov EE, Parovichnikova EN and Savchenko VG. Detection of T-Cell Clonality in Bone Marrow in Peripheral T-Cell Lymphoma, Not Otherwise Specified. Blood 2015; 126 (23): 5020 .

[8] Savage KJ, Chhanabhai M, Gascoyne RD, Connors JM. Characterization of peripheral T-cell lymphomas in a single North American institution by the WHO classification. Ann Oncol. 2004; 15: 1467-1475.

[9] Zhang Y, Wei Xu W, Liu H and Li J. Therapeutic options in peripheral $\mathrm{T}$ cell lymphoma. Journal of Hematology \& Oncology. 2016 (9): 37.

[10] Schmitz N, Trumper L, Ziepert M, et al. Treatment and prognosis of mature T-cell and NK-cell lymphoma: an analysis of patients with T-cell lymphoma treated in studies of the German high-grade non-Hodgkin lymphoma study group. Blood. 2010; 116 (18): 3418-3425.

[11] Mak V, Hamm J, Chhanabhai M, Shenkier T, Klasa R, Sehn LH, Villa D, Gascoyne RD, Connors JM, and Savage KJ. Survival of Patients With Peripheral T-Cell Lymphoma After First Relapse or Progression: Spectrum of Disease and Rare Long-Term Survivors. J Clin Oncol 2013; 31 (16): 1970-1976.

[12] Swerdlow SH, Campo E, Harris NL, Jaffe ES, Pileri SA et al. WHO classification of tumours of haematopoietic and lymphoid tissues. 4th Edition, IARC 2008.

[13] Foss FM, Zinzani PL, Vose JM, et al. Peripheral T-cell lymphoma. Blood. 2011; 117 (25): 6756-6767.

[14] Parrilla Castellar ER, Jaffe ES, Said JW, Swerdlow SH, Ketterling RP, Knudson RA, Sidhu JS, Hsi ED, Karikehalli S, Jiang L, Vasmatzis G, Gibson SE, Ondrejka S, Nicolae A, Grogg KL, Allmer C, Ristow KM, Wilson WH, Macon WR, Law ME, Cerhan JR, Habermann TM, Ansell SM, Dogan A, Maurer MJ, and Feldman AL. ALK-negative anaplastic large cell lymphoma is a genetically heterogeneous disease with widely disparate clinical outcomes. Blood. 2014; 124 (9): 1473-1480.

[15] Edupuganti S, Xie C, Huang J, Chen X, Wey E, and Xie M. Clinicopathological Features of Primary Bone Marrow Mature T and NK Cell Neoplasms. J J Cancer Sci Res. 2016, 2 (7): 046 .

[16] Skarbnik AP, Burki M and Pro B. Peripheral T-cell lymphomas: a review of current approaches and hopes for the future. Frontiers in Oncology. 2013; 3 (138): 1-9.

[17] Broccoli A and Zinzani PL. Peripheral T-cell lymphoma, not otherwise specified. Blood First Edition Paper, 2017; DOI 10.1182/blood-2016-08-692566.

[18] Vose JM. Peripheral T-cell Non-Hodgkin's lymphoma. Hematology/oncology Clinics of North America. 2008; 22 (5): 997-1005. 
[19] The Leukemia \& Lymphoma Society. Peripheral T-Cell Lymphoma Facts. July 2014; page 1-8.

[20] Ma X. Epidemiology of Myelodysplastic Syndromes. Am J Med. 2012; 125 (7 Suppl): S2-S5.

[21] Ria R, Moschetta M, Reale A, Mangialardi G, Castrovilli A, Vacca A, and Dammacco F. Managing myelodysplastic symptoms in elderly patients. Clinical Interventions in Aging. 2009; (4): 413-423.

[22] Luckheeram RV, Zhou R, Verma AD, and Xia B. CD4+T Cells: Differentiation and Functions. Clinical and Developmental Immunology. 2012, Article ID 925135, 12 pages.
[23] Drayton DL, Liao S, Mounzer RH, and Ruddle NH. "Lymphoid organ development: from ontogeny to neogenesis," Nature Immunology, 2006; 7 (4): 344-353.

[24] Monteiro JP, Benjamin A, Costa ES, Barcinski MA, and Bonomo A. Normal hematopoiesis is maintained by activated bone marrow CD4+ T cells. Blood. 2005; 105: 1484-1491.

[25] Broxmeyer HE, Bruns HA, Zhang S, et al. Th1 cells regulate hematopoietic progenitor cell homeostasis by production of oncostatin M. Immunity. 2002; 16: 815-825. 